\title{
RESCATANDO LOS SABORES OCULTOS DEL MAR DEL PERÚ
}

\section{RESCUING THE HIDDEN FLAVORS OF THE SEA OF PERU}

\author{
Jeannette Sampe Palomino \\ Investigadora externa del Instituto de Investigación de Turismo, \\ Universidad de San Martín de Porres, Perú
}

\section{RESUMEN}

Las actividades de pesca de merluza en el Perú dejan un volumen considerable de descartes, constituidos por especies de peces y moluscos que se desechan al mar. Considerando la necesidad de entender la pesquería como parte de un sistema que corre el riesgo de desestabilizarse, se propone rescatar seis especies para que se incorporen a la oferta de restaurantes de alta cocina. Este desafío lo están asumiendo el Instituto del Mar del Perú (IMARPE) el restaurante Costanera 700 y empresas pesqueras de merluza que operan en el país.

Palabras clave: Descartes de pesca, merluza, peces demersales, pesca de arrastre, pesquería sostenible, seguridad alimentaria.

\begin{abstract}
Hake fishing activities in Peru leave an important volumen of discards, that is, several species of fish and mollusks that are returned to the sea. If the fishing activity must be understood as a process in risk of desestabilization, this research proposes a way to rescue six marine species intended to be included within the Haut Cuisine Peruvian restaurants. Thischallenge is being faced by the Peruvian Sea Institut (IMARPE), Restaurant Costanera 700 and some hake fishing companies working in Peru.
\end{abstract}

Key words: Discarded fishing, hake, demersal fishes, trawl fishing, sustainable fishing, food security

\section{Introducción}

Mirador de estrellas, bocón, cocodrilo, buldog, iguana marina, pulpo. Son especies que no se comercializan en el Perú. No se conocen ni se valoran. Pero en las manos correctas pueden tener un mejor destino: no ser descartadas, desechadas, olvidadas. Las especies que se descartan cuando en el Perú se pesca merluza pueden darnos la oportunidad, mediante su disfrute y una buena alimentación, de hacernos partícipes del respeto a la biodiversidad de los océanos. No es poca cosa. 
Los esfuerzos por reducir los descartes de pesca se reflejan en mejores disposiciones legales, pero se requiere un mejor control de todas las actividades relacionadas. Los registros nos hablan de los descartes que se desembarcan; es decir, lo que los pesqueros desean registrar. Para mejorar las políticas al respecto se requiere conocer todo el registro de capturas de todas las embarcaciones, de todos los países.

El presente documento se ocupa de las especies que se descartan en el Perú por la pesca de merluza. Se propone que se utilicen, de manera sostenible, para consumo humano directo y que se puedan encontrar en la oferta de restaurantes. El reto lo están asumiendo el IMARPE, el restaurante Costanera 700 y empresas pesqueras de merluza.

En el pasado los subproductos de la pesca, incluidos los desechos, se consideraban de bajo valor, como un problema que debía eliminarse de la forma más conveniente o descartarse. En los dos últimos decenios se ha registrado una mayor preocupación en el mundo acerca de los aspectos económicos, socialesy ambientales de la utilización óptima de los subproductos de la pesca; así como de la importancia de reducir los descartes y las pérdidas en las fases posteriores a la captura: almacenamiento, elaboración y distribución (FAO, 2012).

\section{El consumo de peces en Lima}

La variedad de frutos de mar que se consume en Lima es amplia, pero las seis más consumidas representan el grueso de la pesca para consumo. Y, según observamos en el siguiente cuadro, la situación es la misma hace muchos años.

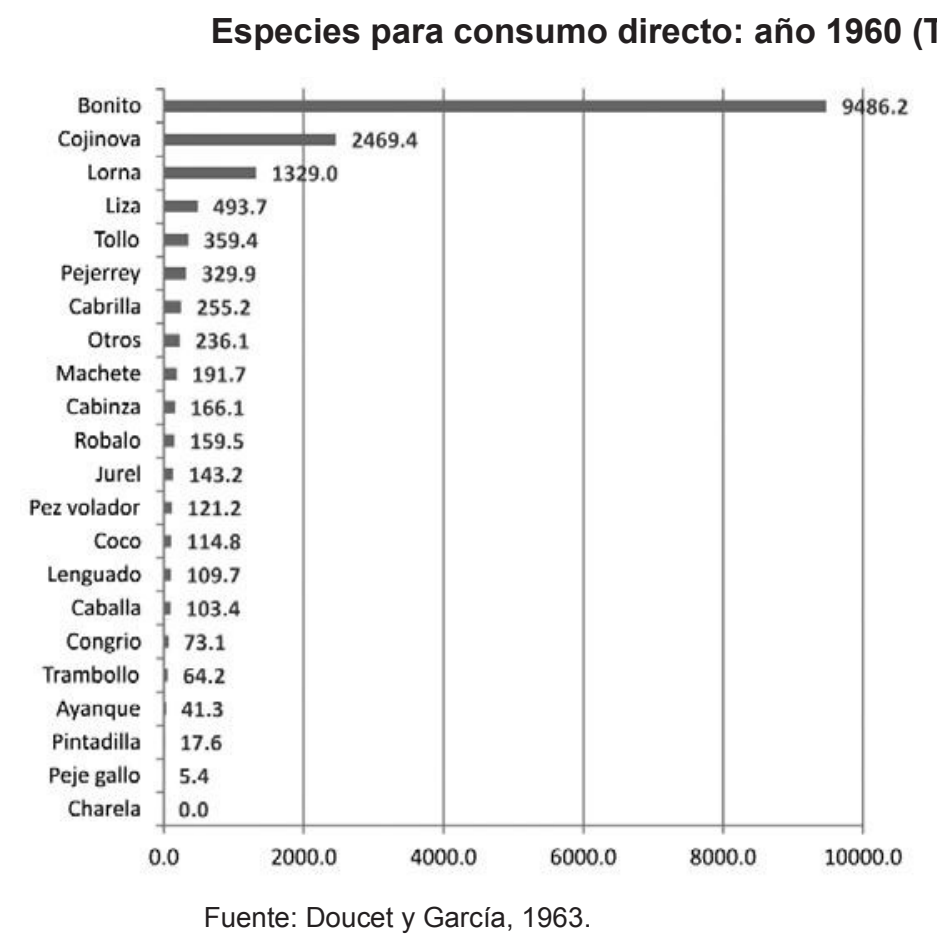


Antes, las especies preferidas del consumidor limeño eran: cojinova, corvina, bonito, liza, tollo y pejerrey, que juntas representaban el 90\% del total consumido en 1962. Según Doucet y García (1963: 20), el crecimiento de la demanda de otras especies es lento: "El público no es muy exigente de la calidad y considera buena la de los productores pesqueros. A medida que el público vaya ganando confianza y familiarizándose con el pescado refrigerado, se creará indudablemente una demanda y tendrán cabida en el mercado limeño especies que en la actualidad son poco conocidas".

La fotografía del consumo de los años sesenta y la de medio siglo después es casi la misma. Los autores acertaban por un lado, pues se ampliaron las especies que se consumían, pero por otro erraron ya que hoy seguimos concentrando el consumo en solo un grupo de frutos de mar. Observemos el siguiente cuadro.

Especies para consumo directo: año 2008 (TN)

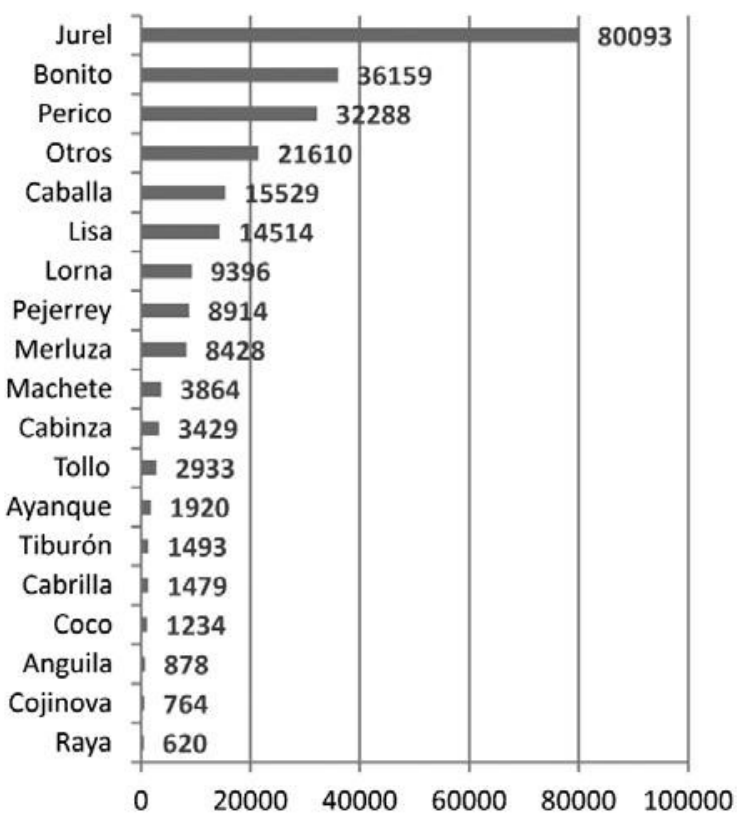

Fuente: Del Carpio y Vila, 2010: 26.

Las seis especies preferidas son: jurel, bonito, perico, caballa, lisa y lorna, que representan el $80 \%$ del consumo total en Lima Metropolitana. La capital peruana, con 8,5 millones de habitantes, consume un promedio de $26,5 \mathrm{~kg}$. per cápita al año. La comercialización de pescado mueve 780 millones de dólares anuales (Del Carpio \& Vila, 2010).

Crecimiento del consumo de pescado frente al de la población - Lima

\begin{tabular}{lccc}
\hline & Año 1960 & Año 2008 & Crecimiento \\
\hline TN de pescado & 16205 & 245545 & 14.2 \\
Población & 828298 & 8445211 & 9.2 \\
\hline
\end{tabular}

Cuadro: Elaboración propia a partir de Del Carpio y Vila, 2010; Doucet y García, 1963; INEI, 2008. 
En el siguiente cuadro se puede observar el crecimiento del consumo, del cual podemos deducir que los limeños están consumiendo más cantidad de pescado, lo que desde el punto de vista nutricional es bueno.

Salvo las de profundidad, las mismas especies se siguen consumiendo hoy, 48 años después. Lo que sí sorprende es que, luego de casi medio siglo, el mar siga brindándonos, y de forma muy generosa, tanta cantidad de peces. También debemos preguntarnos por cuánto tiempo más seguirá siendo sostenible el consumo y la pesca en el Perú. Si bien nos sabemos un país privilegiado por el mar, tan rico y diverso, no nos preocupamos por pensar, como ciudadanos, en la explotación racional de sus recursos (Sampe, 2014, E2).

Otro elemento que nos debe llamar a una profunda reflexión es que entre los recursos que se capturan no siempre están las especies más populares: se pesca especies consideradas no comercializables; es por ello, principalmente, que se desecha y mucho. Sin duda, esta no es una práctica justa, ni para el mar ni para el consumidor.

\section{Los descartes}

Los recursos naturales son considerados por la nación peruana como patrimonio, cuyo uso sostenible es promovido por el Estado. En este marco, el 19 de marzo de 2011 se promulgó el Decreto Supremo Nº 005-2011PRODUCE, que aprobó el reglamento del procesamiento de descartes y residuos de recursos hidrobiológicos, donde se define a los descartes como "aquellos recursos hidrobiológicos adulterados, alterados, descompuestos y contaminado (sic) enteros o por piezas, declarados no aptos para consumo humano directo". En el documento, además, se prescribe que estos descartes y residuos de pesca, generados por la actividad pesquera orientada al consumo humano directo, se deben aprovechar, procesándose por ejemplo en plantas autorizadas de harina de pescado residual, de reaprovechamiento, de ensilado e ictiocompost.

La Comunidad Europea, por otro lado, considera a los descartes como un bien público y norma también su uso en aras de la sostenibilidad de los recursos (Consejo de la Unión Europea, 2013).

La FAO (2011) entiende como descartes de pesca a: "el pescado muerto o el pescado que no puede sobrevivir tras su liberación en vida, que se tira o se pierde" (Directrices P54); mientras que la Marine Stewardship Council brinda la siguiente definición: "Descarte, o captura descartada, es una porción de materia orgánica de origen animal de la captura total que se desaprovecha, o es vertida al mar por cualquier razón. No incluye materiales vegetales ni desechos pos captura, tales como vísceras o entrañas. Los descartes pueden estar muertos o vivos" (MSC, 2012: 42).

Lo curioso es que, en muchos países, los descartes se registran en el desembarque. Así sucede en el Perú. Y, claro, lo que llega a puerto en óptimas condiciones es la especie objetivo de cada navío. Si buscamos merluza como especie objetivo, solo transportaremos con cuidado la merluza u otras pocas especies comunes que se pueden vender con seguridad en tierra. El resto se desecha en el mar o se lleva a puerto para destinarlo a su procesamiento, pero como alimento para animales, pues ya no está apto para el consumo humano directo 
(Sampe, 2014, E1). La investigación de las especies aquí mencionadas ayudará a la formulación de propuestas legislativas sobre manejo de descartes para consumo humano, directo o indirecto, en el Perú.

\section{¿Por qué se producen los descartes de pesca?}

Lo ideal sería que solo se capturara la especie objetivo, pero en la realidad no sucede así, al menos no con la tecnología con la que contamos hoy. En la actualidad, los descartes aparecen a la hora de la pesca. Si bien la forma en que se realiza esta no permite una captura cien por ciento selectiva -tanto en especies como en tamaño-, la razón para que se generen los descartes es económica. Sebastián Losada lo señaló así en un taller realizado en 2002 y, desde la perspectiva de la ONG Green Peace, mencionó algunos motivos para la existencia de los descartes:

- La selección de especies que tienen mayor valor comercial debido a la capacidad limitada de las embarcaciones.

- Las especies que no alcanzan la talla mínima son descartadas por ser ilegales o porque no se pueden comercializar.

- Las especies menos comunes son eliminadas debido a su poco valor comercial (Losada, 2002).

\section{El impacto de los descartes}

El manejo de los descartes no se refiere a lanzarle retos a la pesquería responsable, no se trata de dar discursos sobre sostenibilidad; se trata de entender la pesquería como un conjunto completo e integral de actividades.

Desde un punto de vista económico-productivo, la pesquería se entiende de la siguiente manera: "Está integrada por el conjunto de unidades de pesca (embarcación-tripulación) dedicadas a la extracción de un determinado recurso hidrobiológico (especie o especie y afines), en áreas definidas del mar, con el mismo tipo de embarcaciones, artes y aparejos de pesca" (Espinoza, 1999).

Como señaló Losada (2002) en su presentación en Bruselas, no se trata de gestionar poblaciones de peces solamente, sino de gestionar "el conjunto del ecosistema marino". Esta concepción hará que se perciba de modo distinto a la actividad pesquera. Todos los actores -empresarios pesqueros, gobiernos y sociedad civildeben coadyuvar a desarrollar una actividad pesquera sostenible en el tiempo.

En este sentido, los descartes no son solo especies que se capturaron de manera accidental, sino son especies que, ya capturadas, se deben utilizar con el objetivo de realizar un aprovechamiento integral de la pesca para no alterar el ecosistema marino. Las consecuencias de la captura de los descartes se dan en diferentes niveles. En el nivel de población supone un índice de mortalidad mayor al que se maneja o registra oficialmente. En el nivel trófico y de ecosistema puede provocar un cambio en su estructura, también en la biodiversidad local y en los procesos productivos de las especies, pues dependerá de cuáles especies puedan aprovechar estos 
excedentes "anormales" y cuáles no (Losada, 2002). Pueden afectar asimismo el fondo marino que no está preparado para recibir la cantidad de descartes y mantener su equilibrio ecológico.

La Organización de las Naciones Unidas para la Alimentación y la Agricultura (FAO) señalaba en 1994, en El estado mundial de la pesca y la acuicultura, que los descartes constituían 20 millones de toneladas anuales. Sin embargo, un estudio hecho por Kieran Kelleher para esta entidad estimaba la tasa ponderada anual de los descartes en un $8 \%$ (proporción de la captura descartada sobre la captura total) del total de la pesca. La estimación de este autor, entre 1992 y 2001, es del orden de 7,3 millones de toneladas por año (Kelleher, 2008) ${ }^{1}$. En el Perú, según Kelleher, la pesca de merluza generó descartes de un orden de 15 mil toneladas en el período señalado.

En el siguiente cuadro veremos la cantidad de fauna acompañante de la pesca de merluza. No se contabiliza como descarte, pero nos da una referencia de la cantidad de especies que no se utilizan.

Especies sin uso ni valor comercial capturadas en la pesca de merluza por año (TN)

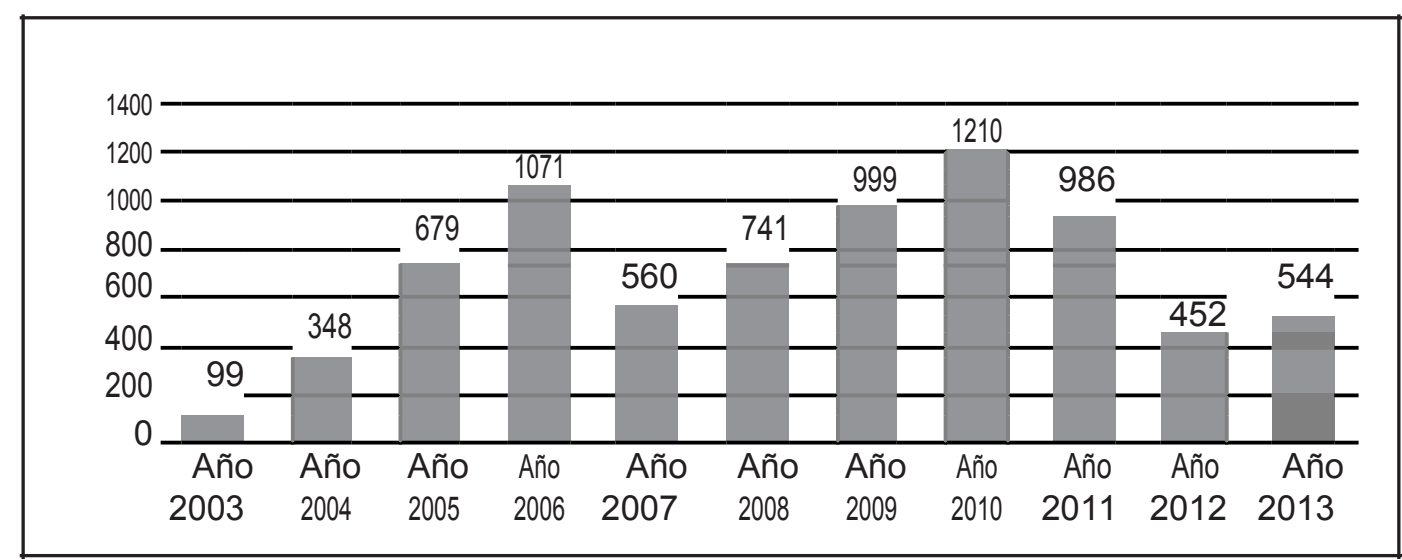

Fuente: IMARPE.

IMARPE nos ha proporcionado también información sobre la cantidad capturada de algunas de las especies que se trabajarán en esta iniciativa de aprovechamiento de los descartes de merluza:

Pesca de algunas especies sin uso ni valor comercial de la pesca de merluza $(\mathrm{kg})$

\begin{tabular}{llllllllllll}
\hline & 2003 & 2004 & 2005 & 2006 & 2007 & 2008 & 2009 & 2010 & 2011 & 2012 & 2013 \\
\hline Bocón & 1689 & 58494 & 72180 & 77315 & 36486 & 28090 & 39023 & 92169 & 59423 & 29153 & 66697 \\
Buldog & 1320 & 25798 & 34729 & 46558 & 23241 & 37989 & 48460 & 62115 & 59834 & 37465 & 66475 \\
Cocodrilo & 1176 & 3335 & 12929 & 29274 & 22492 & 62215 & 39881 & 997 & 301 & 9654 & 8275 \\
Mirador de estrellas & 380 & 151 & 274 & 0 & 6 & 0 & 0 & 0 & 1 & 0 & 0 \\
\hline
\end{tabular}

Fuente: IMARPE.

Para metodología de cálculo de descartes ver Kelleher, 2008: 18. 


\section{Cómo revertir la incidencia de los descartes de pesca}

Losada (2002) sugiere que se pueden revertir los descartes de pesca mediante la disminución del esfuerzo pesquero, siendo más selectivos y definiendo con mayor precisión las artes de pesca para cada especie, así como mejorando el control de la actividad pesquera, no solo desde el desembarque de la especie objetivo e incidental, sino siguiendo toda la actividad que se dé a bordo de las embarcaciones. Esto se complementaría con el cierre de áreas donde se ubiquen especies juveniles imprevistas.

La FAO, a través del documento Directrices internacionales para la ordenación de las capturas incidentales y la reducción de los descartes, de 2011, sugiere algunas medidas para la reducción al mínimo de estos. Si bien se trata de un documento de referencia y de aplicación facultativa, pone en evidencia la necesidad de reglamentar mejor los descartes de pesca. Entre las sugerencias señaladas mencionaremos las siguientes:

- Controlar insumos y producción.

- Mejorar el diseño de las artes de pesca.

- Establecer límites o cuotas de capturas de descartes.

- Los estados deben dar estas medidas con base científica.

- Las medidas deben ser ejecutables e involucrar en su formulación a las partes interesadas.

- Tomar en cuenta que los pescadores colaborarán en la reducción de descartes si esto significa una mejora para sus ingresos (FAO, 2011).

El reglamento europeo, de enero de 2013, al que ya hemos aludido, en su afán de revertir la amenaza a la sostenibilidad que los descartes incontrolados representan, incita a los pescadores a:

- Optimizar la selectividad de las capturas de las operaciones que realizan; es decir, aplicar las técnicas de pesca para cada especie con el fin de evitar la captura de especies no deseadas.

- Incluir todas las operaciones que se realizan en el mar, y no solo la del desembarco que se produce en los puertos. De esta manera se busca racionalizar el manejo de los descartes (Consejo de la Unión Europea, 2013).

Consideramos que un modo de promover el buen manejo de descartes es a través del incentivo a las empresas pesqueras o a los armadores para que consigan la ecocertificación. El aprovechamiento de las especies de los descartes encaja en los principios que rigen los estándares de las certificaciones de pesca sostenible. Tal es el caso de los principios del Marine Stewardship Council (MSC):

- La actividad pesquera debe realizarse a un nivel sostenible para la población de peces. Toda pesquería con certificación debe operar de tal forma que se pueda continuar pescando indefinidamente y sin sobre explotar los recursos.

- Las actividades pesqueras deben gestionarse para mantener la estructura, productividad, función y diversidad del ecosistema del cual depende la pesquería (MSC, 2012). 
En el Perú la certificaciónes aún voluntaria, solo algunas empresas pesqueras están detrás de ella pero, en el futuro, será necesaria. Para lograrla, las empresas deben implementar una serie de acciones, entre ellas el manejo adecuado de los descartes. La certificación de pesca sostenible está en sus inicios, hace falta aún que se adecuen normas, que se realicen investigaciones sobre las especies de descartes y que se generen disposiciones legales que la promuevan.

\section{Fauna acompañante}

Conformada por las especies que se capturan en operaciones dirigidas hacia la pesca de la especie objetivo. En el Perú, en el caso de la pesca de merluza (Merluccius gayi peruanus), que es la especie objetivo, las más frecuentes son: tollo, cabrilla, falso volador, perela, suco, mero, cachema, lorna, lenguado, entre otras (Ministerio de la Producción, 2003).

\section{Zonas de pesca: uso de redes y mallas}

La zona habitual de pesca de merluza en el Perú se ubica en el área marítima comprendida desde el extremo norte del dominio marítimo peruano y el paralelo $06^{\circ} 00^{\prime} 00^{\prime}$ ' S. Las embarcaciones que se dedican a este tipo de pesca son las que usan el sistema de arrastre de fondo o espineleras (Ibíd.).

\section{La oportunidad tomada}

El uso de los descartes es una oportunidad que debería ser aprovechada por los actores involucrados en la explotación de los recursos del mar, quienes tendrían queasociarsepara que su actividad sea sostenible; todo en un marco de acción que los llevará -y a nosotros con ellos- hacia la práctica de una nueva pesquería en el Perú, así como a contribuir con la seguridad alimentaria en el país.

Los descartes pueden ser utilizados en varios terrenos productivos: industria alimentaria pesquera, producción de cosméticos e insumos farmacéuticos, pieles, alimento para animales, fertilizantes y otros (FAO, 2012).

El biólogo Raúl Castillo, director general de investigaciones de recursos demersales y litorales del IMARPE, es un científico convencido de que el uso de los recursos que tenemos en el mar peruano está por debajo de lo que podríamos disfrutar; por ello estudia las especies que no se encuentran en nuestros mercados y promueve su consumo.

El chef Yaquir Sato, responsable de la cocina y del área de investigación y desarrollo del restaurante Costanera 700, afirma que la sostenibilidad no se trata de un discurso sino de una forma de vida; por ello estudia el consumo de especies que puedan ofrecernos calidad y buen sabor sin necesidad de depredar.

Los armadores responsables son aquellas empresas pesqueras que, al respetar los recursos que serían considerados como descarte, podrán darle valor a su quehacer con miras de una ecocertifícación; por ello colaboran con el IMARPE brindando facilidades de transporte, pero en las condiciones que exigen las especies 
marinas, desde el lugar de pesca hasta sus plantas en tierra. Castillo señala al respecto que se ha involucrado a varias empresas pesqueras en este proyecto.

La asociación de estos actores en una cadena positiva hace que imaginemos que es posible una industria pesquera que tome en serio su rol, no solo como comercializadora de recursos, sino en su papel originario, el de alimentar y nutrir responsablemente.

\section{Las especies descartadas}

El caso que nos ocupa se refiere a los descartes de recursos hidrobiológicos que se generan a partir de la pesca de merluza en el Perú.

En mayo de 2013, el Barco Científico de Investigación (BIC) "Humboldt" zarpó con un destino poco usual: un destino gastronómico. Llevaba, además de los habituales científicos y técnicos, un equipo de cocineros encargado de escudriñar entre los descartes de la pesca de merluza y buscar especies que puedan ser consumidas en restaurantes en lugar de ser devueltas al mar sin vida o convertidas en alimento industrial de animales.

Los cocineros hicieron su trabajo, encontraron especies que satisficieron los requerimientos del chef Sato: ser insumos de gran calidad y de mucho sabor.

Las especies revaloradas de los descartes de merluza son demersales y se describen de la siguiente manera:

\section{Pez bocón o tamboreta}

Nombre científíco: Lophiodescaulinaris.

Familia: $\quad$ Lophiidae.

Ubicación: $\quad$ Entre el golfo de California en México y el Callao (18º0’S, $\left.71^{\circ} 12^{\prime} \mathrm{W}\right)$ en Perú, incluyendo las islas Galápagos y las islas Coco. Es una especie bentónica de plataforma, que habita sobre fondos arenosos entre 100 y $190 \mathrm{~m}$. de profundidad. Larvas pelágicas.

Descripción: Boca grande, cabeza con numerosas espinas óseas punzantes, piel suelta y sin escamas. Se le reconoce fácilmente por tener seis manchas blancas en la aleta caudal.

Otros nombres: Bocón de mar, gelatina, murciélago, pez sapo, rape bocón, rape de rabo manchado. En inglés: Spottedtailangler.

Talla:

$15-35 \mathrm{~cm}$.

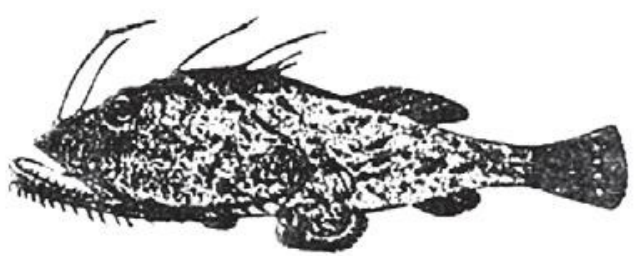

Fuentes: Chirichigno y Cornejo, 2001: 125; Nakaya, K. (Ed.), 2009: 204 


\section{Pez cocodrilo o caballito moro}

Nombre científico: Peristedionbarbiger.

Familia : Triglidae.

Ubicación : Desde la bahía de Panamá hasta Paita en Perú. Es una especie bentónica, que habita sobre fondos arenosos y fangosos.

Descripción : Tiene el cuerpo fusiforme cubierto de placas óseas, arregladas en cuatro filas, con una espina dirigida hacia atrás.

Otros nombres : Acordeón o cocodrilo. En inglés: Crocodilefish.

Talla $\quad: 16 \mathrm{~cm}$.

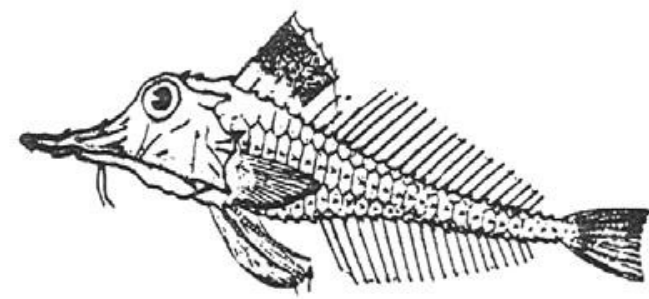

Fuentes: Chirichigno y Cornejo, 2001: 175; Nakaya, K. (Ed.), 2009: 248.

\section{Pez mirador de estrellas o miracielo, perro}

Nombre científico: Astroscopuszephyreus.

Familia : Uranoscopidae.

Ubicación : En Hungtinton Beach (EE.UU.), y entre Mazatlán (México) y Paita (Perú). De modo ocasional se desplaza hasta Chimbote (Perú). Es una especie bentónica, semienterrada sobre fondos arenosos.

Descripción : No se cuenta con información

Otros nombres : Miraestrellas. En inglés: Pacificstargazer.

Talla $\quad: 19-28 \mathrm{~cm}$.

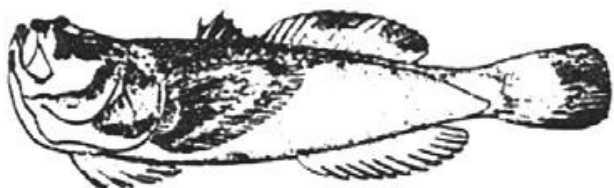

Fuente: Chirichigno y Cornejo, 2001: 19. 


\section{Pez iguana marina o pez lagartija}

Nombre científico: Synodusevermanni.

Familia : Synodontidae.

Ubicación : En Mazatlán, Baja California (México) y a $08^{\circ} 2^{\prime}$ S $, 79^{\circ} 19,9^{`} \mathrm{~W}$ en bahía Chilca (Perú). Es una especie bentónica, que habita sobre fondos arenosos y arenoso-fangosos, entre $25 \mathrm{y}$ $200 \mathrm{~m}$. de profundidad.

Descripción : Cuerpo alargado subcilíndrico y cabeza algo deprimida. Color marrón oscuro con seis a siete manchas oscuras a lo largo de la línea lateral.

Otros nombres : Huavina, lagarto, lagarto garrobo, pez huevo. En inglés: Inottedlizardfish.

Talla $\quad: 17-28 \mathrm{~cm}$.

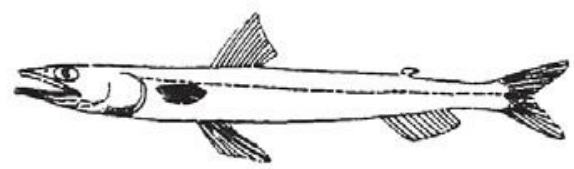

Fuentes: Chirichigno y Cornejo, 2001: 229; Nakaya, K. (Ed.), 2009: 134.

\section{Buldog o astrólogo}

Nombre científico: Kathetostomaaverruncus.

Familia : Uranoscopidae.

Ubicación : En California (EE.UU.), y de Panamá a isla Lobos de Tierra (Perú), e islas Galápagos

(Ecuador). Es una especie bentonerítica semienterrada sobre fondos arenosos y fangosos, hasta $300 \mathrm{~m}$. de profundidad.

Descripción : Cuerpo corto, un poco comprimido, pectoral grande, de color marrón en el dorso y blanco o marrón claro en la cara ventral. Muchas manchas blancas en el dorso y en todas las aletas, excepto las pélvicas.

Otros nombres : Miracielo buldog, miraestrellas. En inglés: Smoothstargazer.

Talla $\quad: 10-20 \mathrm{~cm}$.

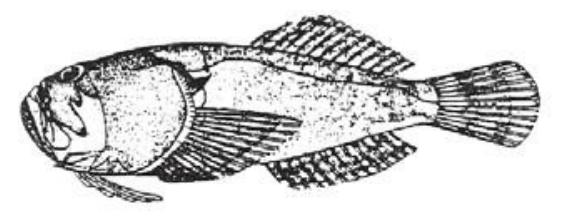

Fuentes: Chirichigno y Cornejo, 2001: 114; Nakaya et al., 2009: 291. 


\section{Pulpo}

Nombre científico: Actualmente IMARPE se encuentra en proceso de clasificación científica de la especie encontrada en el norte del Perú.

Familia : Octopodidae.

IMARPE se encuentra realizando los estudios de esta especie para hacer una determinación científica apropiada.

\section{Los compromisos de los actores involucrados (Sampe, 2014: E1 y E3)}

- IMARPE: Como institución que vela por un mejor conocimiento del mar y de sus recursos hidrobiológicos busca realizar investigaciones más profundas sobre las especies escogidas, con el fin de plantear mecanismos para sugerir normas sobre la utilización de descartes de pesca para consumo humano directo y servir de engranaje entre los actores involucrados en este proyecto.

- Costanera 700: Como restaurante con una trayectoria amplia en manejo de insumos marinos, y reconocido por su alta calidad culinaria, es el soporte gastronómico de esta iniciativa. Ofrecerá dentro de su carta de platos los que se preparen con las seis especies seleccionadas y pondrá a disposición de quienes lo requieran información sobre su uso y recetas.

- Armadores dedicados a la pesca de merluza: Asumen el traslado de las especies en óptimas condiciones, desde las embarcaciones hasta por lo menos el puerto; en un primer momento, sin costo para IMARPE o para el restaurante Costanera 700.

\section{$\operatorname{Recetas}^{2}$}

Como ya se ha dicho, el chef Yaquir Sato busca integrar a su cocina la sostenibilidad y el uso adecuado de las especies marinas. Convencido de que el respeto por la biodiversidad marina se manifiesta a través de acciones cotidianas y concretas, ha asumido la tarea de introducir en la carta del restaurante Costanera 700 especies de descartes de pesca. A continuación se presentan deliciosas y sencillas recetas, todas creadas por Sato.

Cabe señalar que las bases descritas en las recetas son preparaciones que se hacen con anticipación, pasteurizadas y esterilizadas. Se pueden preparar en casa con los insumos que se indican para cada caso.

\section{Sudado de pez bocón, buldog o mirador de estrellas}

\section{Ingredientes}

25 g de cebolla

$25 \mathrm{~g}$ de tomate

Para revisar otros trabajos sobre recursos hidrobiológicos y gastronomía, ver Noriega y Zapata, 2008. 
$99 \mathrm{ml}$ de base de sudado

$15 \mathrm{ml}$ de fondo de pescado

$150 \mathrm{~g}$ de filete de pescado

$2 \mathrm{~g}$ de culantro

\section{Base del sudado}

Aceite, cebolla roja, ajo, pasta de ají panca, achiote, vino blanco, pisco, chicha de jora, fumet, glutamato monosódico, tomate y kion.

\section{Preparación}

En un wok saltear las cebollas y tomates con un chorrito de aceite. Agregar la base del sudado y remover. Añadir el filete y el caldo, reducir durante diez minutos. Servir y terminar con culantro picado.

\section{Parihuela de pez bocón o buldog}

\section{Ingredientes}

$156 \mathrm{ml}$ de base de parihuela

$35 \mathrm{ml}$ de fondo de pescado

1 cucharadita de wakame

1 cangrejo

3 choros

$150 \mathrm{~g}$ de filete de pescado

1 porción de mix de mariscos

$5 \mathrm{~g}$ de chuño

\section{Base de parihuela}

Aceite, cebolla roja, ajo, pasta de ají panca, achiote, vino blanco, pisco, chicha de jora, fumet, glutamato monosódico, tomate y kion.

\section{Preparación}

Colocar en una sartén la base de parihuela. Añadir el fondo de pescado, el filete, el cangrejo y el choro. Hervir durante diez minutos. A los ocho minutos incorporar el mix de mariscos. Terminar con el chuño diluido en un poco de agua. Servir. 


\section{Oilmushi de pez bocón}

\section{Ingredientes}

1 kilo de pescado bocón

$810 \mathrm{ml}$ de base Costanera

$5 \mathrm{ml}$ de aceite de ajonjolí

2 cucharadas de cebolla china picada

1 cucharada de kion cortado en

juliana Base Costanera

Kion o jengibre, azúcar, glutamato monosódico, sillao o salsa de soya, agua, ajo.

\section{Preparación}

Colocar el pescado en una fuente y agregarle la base Costanera. Llevar a horno precalentado o al vapor durante diez minutos. Servir, colocándole encima la cebolla china picada y el kion en juliana. Terminar rociándole el aceite de ajonjolí previamente calentado en una sartén hasta el punto de que humee.

\section{Batayaki de pez cocodrilo}

\section{Ingredientes}

12 pescados

1 receta de sazonador de batayaki

1 cucharada de mantequilla con sal

Sazonador de batayaki

$2.5 \mathrm{~g}$ de ajo

$2 \mathrm{~g}$ de sal

3 g de glutamato monosódico

$1 \mathrm{~g}$ de pimienta

\section{Preparación}

Condimentar el pescado con el sazonador de batayaki. En una plancha de hierro untar la mantequilla y llevarla al fuego hasta que se derrita. Colocar los pescados y cocinarlos. A los tres minutos darles vuelta y servir. 
Teriyaki de pez cocodrilo, pulpo e iguana marina

\section{Ingredientes}

$150 \mathrm{~g}$ de pescado o pulpo o iguana

$30 \mathrm{~g}$ de pimiento en trozos

$30 \mathrm{~g}$ de apio en trozos

$5 \mathrm{ml}$ de base de teriyaki

Base de teriyaki

Agua, azúcar, mirin, hondashi, salsa de soya, pata de pollo, katsuobushi y kombu.

\section{Preparación}

Insertar los trozos de pescado en una brocheta alternando con el apio y el pimiento. Bañar con salsa teriyaki y llevarla a cocción en una plancha o parrilla. A los tres minutos darle vuelta y terminar de cocinar.

\section{Anticucho de pez cocodrilo, pulpo e iguana marina}

\section{Ingredientes}

$150 \mathrm{~g}$ de pescado

$75 \mathrm{~g}$ de salsa de anticucho

$30 \mathrm{~g}$ de apio en trozos

$30 \mathrm{~g}$ de pimiento en trozos

80 g de papa

30 g de choclo

\section{Salsa de anticucho}

Pasta de ají panca, vinagre tinto, shoyu, sal, glutamato monosódico, pimienta blanca, comino, ajo, orégano seco y azúcar.

\section{Preparación}

En una brocheta insertar los trozos de pescado alternando con el apio y el pimiento. Untar con la salsa de anticucho y llevar a cocción en una plancha o parrilla. A los tres minutos darle vueltas y terminar de cocinar. Servir acompañado de papa y choclo sancochados.

\section{Recomendaciones para la manipulación del pulpo}

Para una óptima cocción haga lo siguiente: luego de extraer las vísceras colocarlo en una bandeja y agregarle sal. Friccionarlo y empujarlo contra la bandeja, repetir hasta que expulse toda la baba. Lavar con abundante agua. Hervir agua en una olla e introducir, por cinco segundos, la mitad del pulpo para que las puntas de los brazos se enrollen, luego introducir las dos terceras partes, repetir hasta que se enrollen por completo. Cocinar por cuarenta minutos y escurrir bien. 


\section{Conclusiones}

- La preocupación por un buen manejo de descartes de pesca se da a nivel mundial y las normas que se están promulgando contribuyen a lograr un mejor control.

- La normatividad sobre el uso de descartes debe tomar en cuenta no solo la actividad de pesca de determinada especie, sino el ecosistema marino como un complejo.

- La normatividad en el Perú sobre descartes de pesca es muy restrictiva a la concepción de desecho. Mediante la investigación de especies de descarte en la pesquería de la merluza se puede abrir una oportunidad para su aprovechamiento en el consumo humano directo.

- La asociación entre IMARPE, el restaurante Costanera 700 y las empresas pesqueras es pionera en el aprovechamiento de descartes. Representa un reto para que el Perú asuma con liderazgo el camino hacia la nueva pesquería, mediante el manejo correcto de recursos hidrobiológicos. 


\section{Referencias}

Chirichigno, N. \& Cornejo, M. (2001). Catálogo comentado de los peces marinos del Perú. Callao: Instituto del Mar del Perú (IMARPE).

Consejo de la Unión Europea (2013). Reglamento de procesamiento de descartes y/o residuos de recursos hidrobiológicos, 25 de enero.

Del Carpio, L. \& Vila, B. (2010). El mercado de productos pesqueros en la Región Metropolitana de Lima. Serie: El mercado de pescado en las grandes ciudades latinoamericanas. Montevideo: Infopesca.

Doucet, W. \& García, J. (1963). El mercadeo del pescado en Lima: preferencias de los consumidores. Informe 19. Callao: Instituto de Investigación de los Recursos Marinos.

Espinoza, C. (1999). Manual de legislación pesquera en el Perú. Lima: Muñiz, Forsyth, Ramírez, Pérez-Taiman \& Luna Victoria Abogados.

FAO (2011). Directrices internacionales para la ordenación de las capturas incidentales y la reducción de los descartes. Roma: Organización de las Naciones Unidas para la Alimentación y la Agricultura.

FAO (2012). El estado mundial de la pesca y la acuicultura. Roma: Organización de las Naciones Unidas para la Alimentación y la Agricultura.

IMARPE (2014). Información directa recibida del Director de IMARPE.

INEI (2008). Censos Nacionales de Población y Vivienda 1940, 1961, 1972, 1981, 1993 y 2007. Instituto Nacional de Estadística e Informática. Recuperado de http://www.inei.gob.pe/estadisticas/indice-tematico/poblacion-y-vivienda/

Kelleher, K. (2008). Descartes en la pesca de captura marina mundial. Una actualización. Documento Técnico de Pesca $\mathrm{N}^{\circ} 470$. Roma: FAO.

Losada, S. (2002). Descartes en la industria pesquera: una perspectiva desde las ONG. Presentación en el taller "Descartes en aguas comunitarias de especies comerciales" Bruselas.

MSC (2012). Requisitos para la Certificación MSC. London: Marine Stewardship Council.

Ministerio de la Producción (2003). Decreto Supremo $N^{\circ}$ 016-2003-PRODUCE. Se aprueba el Reglamento del ordenamiento pesquero del recurso merluza, 30 de mayo.

Ministerio de la Producción (2007). Resolución Ministerial $N^{\circ} 005-2007-P R O D U C E$. Amplían área de pesca del recurso merluza, 10 de enero.

Ministerio de la Producción (2011). Decreto Supremo $N^{\circ}$ 005-2011-PRODUCE. Se aprueba el Reglamento de procesamiento de descartes y/o residuos de recursos hidrobiológicos, 19 de marzo.

Nakaya, K. (Ed.) (2009). Deep-sea Fishes of Peru. Libro de Peces de aguas profundas del Perú. Tokyo: Japan Deep Sea Trawlers Association.

Noriega, C. \& Zapata, S. (2008). Recursos hidrobiológicos en la gastronomía peruana. Lima: Universidad de San Martín de

Porres. Sampe, J. (2014). E1. Entrevista del 18 de enero a Raúl Castillo.

Sampe, J. (2014). E2. Entrevista del 7 de febrero a Norma Chirichigno.

Sampe, J. (2014). E3. Entrevista del 20 de enero a Yaquir Sato. 
